$\Rightarrow$ CANCER GENOMICS

\title{
Keeping score with immunotherapy
} response

Immunotherapy has a substantial impact on the outcome of some cancers, but its potential is limited because only a minority of patients respond to treatment. Thus, a major motivation in the field is to identify 'signatures' that might predict with high confidence those tumours that can be treated successfully. In this study, Charoentong et al. introduce such a measure for solid tumours - the immunophenoscore. They demonstrate that the score is a strong predictor of melanoma response to two different checkpoint blocker immunotherapy drugs.

Using a strategy designed for a previous analysis of colorectal cancer (CRC), the authors performed extensive bioinformatic analyses of genomic and transcriptomic (RNA sequencing) data from The Cancer Genome Atlas (TGCA) for more than 8,000 samples across 20 solid cancer types. The data are available through a web-based searchable database, The Cancer Immunome Atlas (TCIA), and provide information on a number of important immunogenomic characteristics. These include the cellular composition of tumour infiltrating lymphocytes (TILs), to inform on potential checkpoint blocker immunotherapies; cancer antigen profiles (both neoantigens and cancer germline antigens (CGAs)), to inform on potential vaccine targets; and tumour heterogeneity and clonality as an indication of the proportion of tumour cells that might be susceptible to treatment.

Using a machine learning approach, the authors identified a minimal panel of genes whose expression levels had the greatest prognostic and predictive power across all 20 cancers. The chosen genes could be grouped according to whether they acted as biomarkers for immune effector cells, immune suppressor cells, HLA molecules or other immunomodulators. The TCIA web tool enables data for each sample to be visualized as an immunophenogram and a composite score - the immunophenoscore - can be determined. The immunophenoscore is a measure of the overall immunogenicity of an individual tumour, with a higher score indicative of better prognosis and better response to immunotherapy. As validation, immunophenoscores were able to stratify two clinical cohorts into responders and non-responders to treatment of melanoma with either of two different checkpoint blockers.

The TCIA is an important resource for researchers, enabling them to formulate and test hypotheses that examine the impact of the cancer genome on immune response, particularly with regard to tumour progression and treatment. Although the data set is currently limited to 20 solid cancers and the immunophenoscore has been validated only for melanoma, the overall strategy should serve as a framework for other cancers. Importantly, the immunophenoscore is a potential step toward improved immunotherapy based on personal genomics.

Dorothy Clyde

ORIGINAL ARTICLE Charoentong, P. et al.

Pan-cancer immunogenomic analyses reveal genotype-

immunophenotype relationships and predictors of response to checkpoint blockade. Cell Rep. 18, 248-262 (2017) FURTHER READING Hackl, H. et al. Computational genomics tools for dissecting tumour-immune cell interactions. Nat. Rev. Genet. 17, 441-458 (2016)

WEB SITE

The Cancer Immunome Atlas: https://tcia.at 